\title{
Antioxidative and neuroprotective effects of Leea macrophylla methanol root extracts on diazepam-induced memory impairment in amnesic Wistar albino rat
}

Sakia Ferdousy ${ }^{1}$, Md Atiar Rahman ${ }^{1 *}$, Md Mamun Al-Amin², Jannatul Aklima ${ }^{1}$ and J. M. Kamirul Hasan Chowdhury ${ }^{1}$

\begin{abstract}
Background: A neurological disorder is becoming one of the major health problems worldwide. Synthetic drugs for neurological disorders often produce unwanted effects while traditional plant- derived drugs offer a unique strategy in combating the neurological diseases. This study investigated how the antioxidative properties of Leea macrophylla root extracts integratively help as neuroprotective and antiamnesic in animal model.

Methods: Leea macrophylla root methanol extract (LM) was assessed by open field test, hole cross test, elevated plus maze test and thiopental sodium induced sleeping time test for screening neuropharmacological effect on Wistar albino rats using the LM at $100 \& 200 \mathrm{mg} / \mathrm{kg}$ body weight. Antiamnesic effect of LM was studied on diazepam induced anterograde amnesia in Wistar albino rats. Morris water maze test was performed to evaluate whether the learning and memory of rats are impaired by amnesia produced by diazepam. The antioxidative enzymes superoxide dismutase, catalase, glutathione peroxidase and oxidative biomarkers malondialdehyde, nitric oxide, advanced oxidation protein product from the hippocampus of decapitated animals were measured to assess the antioxidative potential of the extract.
\end{abstract}

Results: LM was found to show significant $(p<0.05)$ reduction in locomotor activity and increase in the duration of sleeping of animals. Morris water maze test showed that animals treated with LM significantly $(p<0.05)$ decreased the mean escape latency compared to animals in diazepam group. LM was also found to reduce the content of malondialdehyde, nitric oxide and advanced oxidation protein product and to increase the activities of superoxide dismutase, catalase and glutathione peroxidase in hippocampus.

Conclusion: Our studies suggest that antioxidative capacity of LM in hippocampus may augment the antiamnesic effects in anterograde amnesia.

Keywords: Leea macrophylla, Diazepam, Amnesia, Antioxidant, Hatikana

Abbreviations: LM, Leea macrophylla root methanol extract; BW, Body weight; CNS, Central Nervous system; NC, Normal control; PC, Positive control; EPM, Elevated plus maize; GABA, y amino butyric acid; ROS, Reactive oxygen species; SOD, Superoxide dismutase

\footnotetext{
*Correspondence: atiar@cu.ac.bd

'Department of Biochemistry \& Molecular Biology, University of Chittagong,

Chittagong 4331, Bangladesh

Full list of author information is available at the end of the article
} 


\section{Background}

Human neurological disorders have posed an ever increasing threat to public health care. Amnesia is a severe disruption of memory without deficits in intelligence, attention, perception or judgment. Anterograde amnesia leads to a partial or complete inability to recall the recent past eventually result from damage to the hypothalamus and thalamus and the surrounding cortical structures. Recent studies suggested that oxidative stress is linked to several neurological disorders as well as amnesia [1]. Therefore, investigation of therapies is an urgent which ameliorates the neurological disorders and amnesia by relieving oxidative stress.

Current therapeutic options, such as interventional procedures, surgery, and synthetic drug, are limited in their ability to improve neural function because they fail to repair damaged neurons or improve neural regeneration [2]. They sometimes cause sedation and myorelaxation [3]. Due to such failure of therapies in neuronal damage repair and severe adverse effects of antiamnesic drugs, alternative treatments as herbal or plant-derived drugs are increasingly being used to alleviate the affective disorders [4]. Similar therapeutic effects with lesser side effects are considered as added advantage of these drugs. Especially the natural antioxidative flavonoids possessing anxiolytic effect is the prime choice as neuroprotective and antiamnesic [5].

Leea macrophylla (Hatikana) is a herbaceous shrub primitively identified as Indian habitat also known as Hastikarnapalasa, Hathikana or Hatkana (Elephants ear) belonging to Leeaceae family. It is around $90 \mathrm{~cm}$ in height with switchy branches and perennial tuberous roots. It is distributed to the relatively hotter parts of India, from the Eastwards of Ganges, Bihar, Bengal, Assam, the Terai and its contiguous plains and also in the western India from Konkan south-wards [6]. In Bangladesh, the plant is found in Chittagong, Rajshahi, Natore and other northern parts of the country [7]. The plant has been observed useful in cancer [8], arthritis, goiter and tetanus [9], urolithiasis [10], hepatic damage [11] and microbial infection [12]. This research investigated whether antioxidative effects of LM contribute as neuroprotective as well as antiamnesic in diazepaminduced animal model.

\section{Methods}

\section{Collection and identification of plant materials}

The plant material, Leea macrophylla roots were collected from cultivated area of Bangladesh Council of Scientific and Industrial Research (BCSIR), Rajshahi in March, 2014. The plant sample was identified and taxonomically authenticated as Leea macrophylla by Dr. Sheikh Bokhtear Uddin, Professor, Department of Botany, University of Chittagong. A sample specimen has been preserved as the accession number ACCU-2011/07 for future reference.

\section{Preparation of plant extract}

The roots of Leea macrophylla were washed and chopped into small pieces, oven-dried at $45{ }^{\circ} \mathrm{C}$. Dried materials were ground into powder by mechanically prepared Willy mill. Resulting powder (600 g) was then defatted with n-hexane extraction. Defatted materials were dissolved in $99 \%$ distilled methanol and left for 24-72 h at room temperature $(23 \pm 0.5){ }^{\circ} \mathrm{C}$ with occasional stirring. The supernatant for repetitive extraction was filtrated through cheesecloth and Whatman filter paper no 1 and the filtrates were collected to get dried under reduced pressure (temperature below $50{ }^{\circ} \mathrm{C}$ ) using rotatory vacuum evaporator (RE200, Bibby sterling Ltd, UK). The concentrated extracts were collected in a Petri dish and allowed to air dry for complete evaporation. Black-green semisolid crude (39 g, yield $6.5 \%$, $\mathrm{W} / \mathrm{W})$ was preserved at $4{ }^{\circ} \mathrm{C}$ for further use.

\section{Experimental animals and maintenance}

Six to seven weeks old adult Wistar albino rats average weight $150.66 \pm 5.87 \mathrm{~g}$ of either sex were selected for neuroprotective activities. The rats were procured from the animal house of Bangladesh Council of Scientific and Industrial Research (BCSIR), Chittagong-4220, Bangladesh. They were healthy and acclimatized for a week to standard laboratory conditions before the starting the experiment. Throughout the experimental period, the animals were housed individually in polycarbonated cages $\left(38 \times 23 \times 10 \mathrm{~cm}^{3}\right)$ at room temperature $(23 \pm 2){ }^{\circ} \mathrm{C}$, relative humidity (60-70\%) and were exposed to $12: 12 \mathrm{~h}$ light: dark cycle. They were supplied with a standard pellet diet and water ad libitum. All animal experimentations were maintained and carried out with the guidelines of Animal Ethics Review Board of Faculty of Biological Sciences, University of Chittagong (approval no. AERB/ FBS/UC/05, 2015).

\section{Drugs and chemicals}

Tween-80 was purchased from Scharlab S. L., Spain. Diazepam was kindly donated by Opsonin Pharmaceuticals Ltd., Bangladesh. Thiopental sodium (Genisia, Square pharmaceuticals Ltd.) was purchased from local market. All other chemicals used in this research were of analytical reagent grade until unless specified with additional reference.

\section{Acute toxicity test and dosage adjustment}

Acclimatized Wistar albino rats maintained in the mentioned laboratory condition were used for acute toxicity study. Five animals received a single oral dose of $0.5,1.0$, 1.5, and $2.0 \mathrm{~g} / \mathrm{kg}$ Body Weight (BW) of LM. Animals 
were kept over-night fasting prior to administration the LM. After administration, food was withheld for further 3 to $4 \mathrm{~h}$. Individual animal was kept in close observation during the first $30 \mathrm{~min}$ after dosing, periodically first $24 \mathrm{~h}$ (special attention for the first $4 \mathrm{~h}$ ), thereafter for a period of 3 days to record the delayed toxicity. Once daily cage side observation including changes in skin and fur, eyes and mucous membrane, respiratory and circulatory rate, autonomic and central nervous system (CNS) changes were observed. The effective therapeutic dose was taken as one tenth of the median lethal dose $\left(\mathrm{LD}_{50}>2.0 \mathrm{~g} / \mathrm{kg}\right)$ [13]. This is an established and widely used model supported by a number of researchers $[14,15]$.

\section{Experimental procedure for neuropharmacological tests}

To investigate neuropharmacological activity open field test, hole cross test, elevated plus maze (EPM) test and thiopental sodium-induced sleeping time tests were performed. For each of these tests, the animals were divided into four groups' namely normal control (NC), positive control (PC) and treatment control Leea macrophylla root methanol extract $100 \mathrm{mg} / \mathrm{kg}$ BW (LM100) and treatment control Leea macrophylla root methanol extract (LM200) group. Each group contains six to eight rats. $\mathrm{NC}$ group received oral administration of vehicle (1\% Tween 80 in water). PC group received Diazepam (reference standard) at the dose of $1 \mathrm{mg} / \mathrm{kg}$ $\mathrm{BW}$, intraperitoneally. The dose of diazepam for the assessment of neurological behavior is selected from literatures [16, 17]. LM100 and LM200 group received LM orally at 100 and $200 \mathrm{mg} / \mathrm{kg}$ BW respectively. Neuropharmacological and behavioral tests are graphically presented in Fig. 1.

\section{Open field test}

The experiment was performed according to the method described by Gupta et al. [18]. The open field apparatus consisted of a wooden floor of half square meter with a series of squares each alternatively colored black and white. The apparatus have a wall of $40 \mathrm{~cm}$ height. Animals were treated with NC, PC, LM100 \& LM200 and were placed in the middle of the open field. The numbers of squares visited by the animals were counted. It was counted for $3 \mathrm{~min}$ at $0,30,60,90$ and $120 \mathrm{~min}$ following the treatments. The neurological apparatuses were thoroughly cleaned with $70 \%$ isopropanol after each trial.

\section{Hole-cross test}

The method was carried out as described by Takagi et al. [19]. For this experiment, a cage was used having a size of $30 \times 20 \times 14 \mathrm{~cm}$ with a fixed partition in the middle. A hole of $3 \mathrm{~cm}$ diameter was made at a height of $7.5 \mathrm{~cm}$ in the center of the cage. After treatment with NC, PC, LM100 and LM200 the animals were allowed to cross the hole from one chamber to another and the numbers of passage of a rat through the hole from

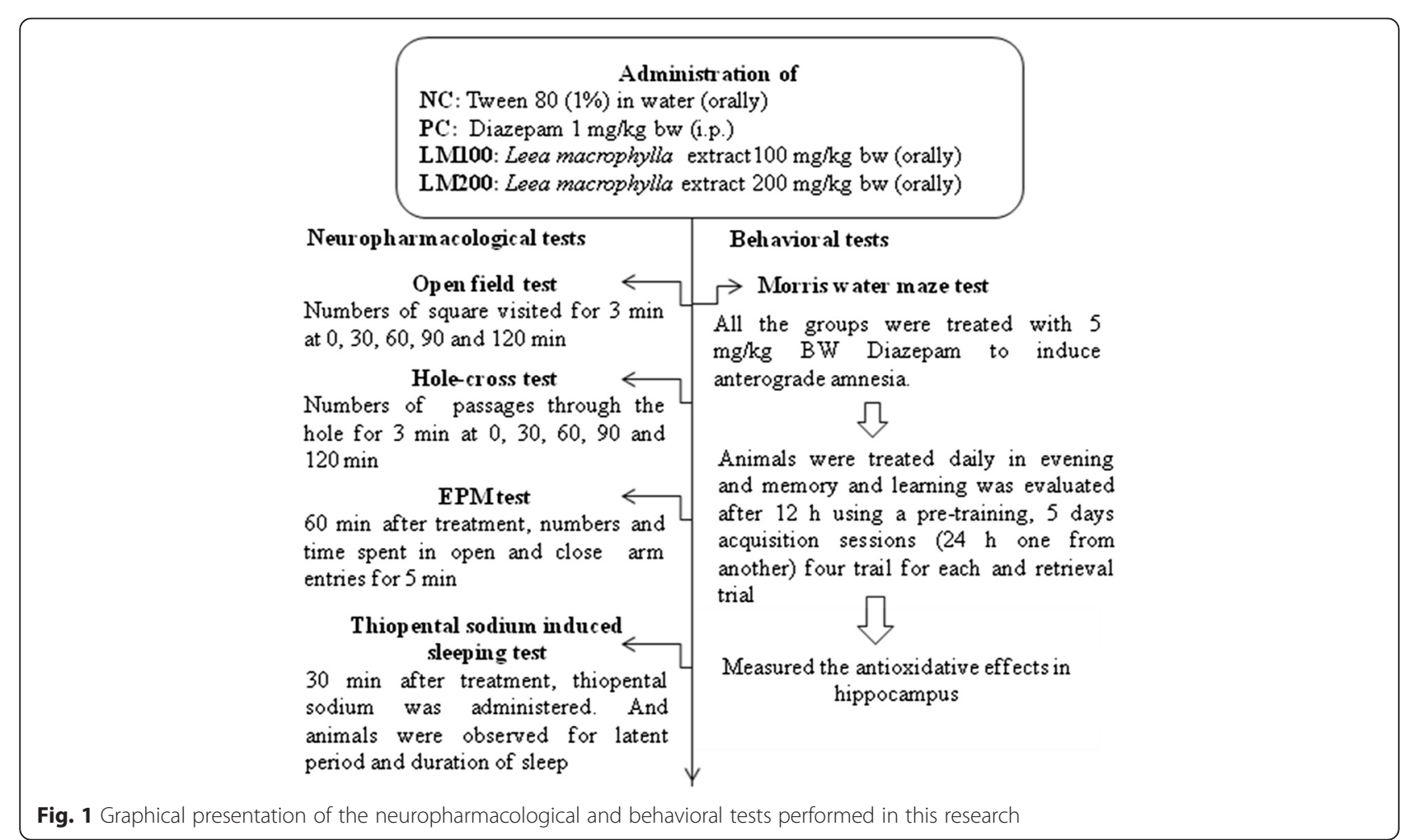


one chamber to other was counted. It was counted for a period of $3 \mathrm{~min}$ at $0,30,60,90$ and $120 \mathrm{~min}$ following the treatments.

\section{EPM test}

The EPM test was carried out by the protocol introduced by Walf and Frye with slight modification [20]. The EPM apparatus consists of two open arms $(5 \times$ $10 \mathrm{~cm})$ and two closed arms $(5 \times 15 \mathrm{~cm})$ radiating from a platform $(5 \times 5 \mathrm{~cm})$ to form a plus sign figure. The apparatus was situated $40 \mathrm{~cm}$ above the floor. The open arms edges were $0.5 \mathrm{~cm}$ in height to keep the rats from falling and the closed-arms edges were $15 \mathrm{~cm}$ in height. Sixty min after administration of the test extract and drugs, each animal was placed at the center of the maze facing one of the enclosed arms. During a $5 \mathrm{~min}$ test period, the number of open and enclosed arms entries and the time spent in open \& enclosed arms was recorded. Entry into an arm was defined as the point when the animal places all four paws onto the arm. The procedure was conducted in a sound attenuated room; observations made from an adjacent corner.

\section{Thiopental sodium induced sleeping time test}

The method was carried out as described by Fujimori and Cobb [21]. Briefly, 30 min after NC, PC, LM100 and LM200 administration, thiopental sodium (at $40 \mathrm{mg} / \mathrm{kg}$ body weight dose) was administered to each rat to induce sleep. The animals were observed for the latent period (time between thiopental administration and loss of righting reflex) and duration of sleep (i.e. time between the loss and recovery of righting reflex) induced by thiopental sodium.

\section{Investigation for anti-amnesic effect}

The experiment was carried out as described by Wang et al. [22]. The animals were divided as earlier. NC group received distilled water only. PC group was treated with diazepam at $5 \mathrm{mg} / \mathrm{kg}$ body weight orally. LM100 group received diazepam at $5 \mathrm{mg} / \mathrm{kg}$ BW and $\mathrm{LM}$ at the doses of $100 \mathrm{mg} / \mathrm{kg} \mathrm{BW}$. LM200 group received diazepam at $5 \mathrm{mg} / \mathrm{kg}$ BW and LM at the doses of $200 \mathrm{mg} / \mathrm{kg} \mathrm{BW}$. In this study, diazepam was administered to induce anterograde amnesia in animals and the dose of diazepam was selected from literature [23]. The study was designed to examine anti-amnesic effect of test sample on diazepam induced amnesia. The animals were treated daily in the evening. After $12 \mathrm{~h}$, the learning and memory of rats was evaluated.

\section{Acquisition and retrieval trials using Morris water maze}

The Morris water maze is one of the most widely used tasks in behavioral neuroscience for studying the psychological processes and neural mechanisms of spatial learning and memory, which are major functions of the hippocampus $[24,25]$. The water maze was developed by Richard Morris and placed navigation in the water maze is now often used as a general assay of cognitive function, for example for testing the impact of various disturbances of the nervous system e.g. animal models of stroke, aging, neurodegenerative disease, or the potential impact of novel therapeutic drugs [26]. It has, therefore, been extensively used for the evaluation of spatial memory deficit induced by Benzodiazepines [27]. This model suitably combines the evaluation of both spatial memory and locomotor activity. Therefore, it can be used for the evaluation of drugs that not only affect spatial memory but also impair locomotor activity.

The water maze consisted of a circular tank, $110 \mathrm{~cm}$ in diameter and with a wall $40 \mathrm{~cm}$ in height. A white platform $(10 \mathrm{~cm}$ in diameter) was placed inside, and the tank was filled with water $\left(22 \pm 1{ }^{\circ} \mathrm{C}\right)$, until the top of the platform was submerged $1 \mathrm{~cm}$ below the water's surface. Before beginning acquisition training, each rat received a pre-training session that consisted of placing the rat on the platform where it had to stay at least $15 \mathrm{~s}$, followed by a $30 \mathrm{~s}$ swimming period, and ending by several trials of climbing onto the platform until each subject was able to climb without help. This nonspatial procedure was required to avoid confusion between procedural aspects of the task and subsequent spatial performance [28]. The following day, the rats were given five acquisition sessions with $24 \mathrm{~h}$ distance one from another, each acquisition consisted of four trials per day with an intertrial interval of $10 \mathrm{~min}$. Throughout the course of this acquisition period, the hidden platform remained in the same fixed position for all rats. In the present study, quadrant Q3 was designated as the target quadrant. In the beginning of each trial, the rats were released with its head facing the wall at one of four points located along the perimeter of the maze, arbitrarily designated as N, S, E, and W. The order of the starting points was determined randomly, and each starting point was used only once per session. Once a rat located the platform, it was allowed to remain there for $30 \mathrm{~s}$ before being removed from the tank. If a rat failed to locate the platform within $60 \mathrm{~s}$, it was manually guided to it. Mean escape latency (the time between being placed in the water and finding the hidden platform) was calculated which is considered as an index of acquisition. After five sessions of acquisition training, a subsequent trial was conducted in which the platform was removed from the pool and the rat had to swim again for $60 \mathrm{~s}$ with no opportunity to escape. This is the retrieval trial study which evaluated how well the animals had learned and consolidated the platform location during the 5 days of training. The percentage of time spent 
in target quadrant by the animal was calculated. The time spent by the animal in the target quadrant (Q3) while searching for the hidden platform was recorded as an index of retrieval.

\section{Assays of Superoxide dismutase (SOD), Catalase (CAT), Glutathione peroxidase (GSH-Px), Malondialdehyde (MDA) and advanced oxidation of protein product (AOPP) estimation}

Twelve hours after evaluation of learning and memory, the rats were sacrificed through chloroform anesthesia and transcardial perfusion with ice-cold normal saline $(10 \mathrm{~mL} / 10 \mathrm{~g} \mathrm{BW})$. The entire intact brain was carefully removed and placed on an ice-chilled dish for cleaning. The hippocampus was rapidly removed and weighed. Then the hippocampus were homogenized in 4 volumes of $0.1 \mathrm{~mol} / \mathrm{L}$ ice phosphate buffer $(\mathrm{pH} 7.4)$ and centrifuged at $20,000 \times \mathrm{g}$ for $30 \mathrm{~min}$ at $4{ }^{\circ} \mathrm{C}$. SOD activity in the hippocampus was determined by the method of Misra and Fridovich [29] based on auto oxidation of epinephrine. Briefly, an aliquot of $0.25 \mathrm{ml}$ ice cold chloroform was added to $0.1 \mathrm{ml}$ of supernatant followed by adding $0.15 \mathrm{ml}$ ice cold ethanol. The mixture was centrifuged at $3000 \mathrm{rpm}$ for $10 \mathrm{~min}$ at $4{ }^{\circ} \mathrm{C}$. Then $0.2 \mathrm{ml}$ of supernatant was taken and $1.5 \mathrm{ml}$ carbonate buffer, $0.5 C C$ EDTA, and $0.8 \mathrm{ml}$ distilled water was added. Reaction was started by adding $0.4 \mathrm{ml}$ epinephrine. Change in absorbance $\Delta \mathrm{OD} / \mathrm{min}$ at $480 \mathrm{~nm}$ was read for $3 \mathrm{~min}$. The results were expressed in terms of $\mathrm{nmol} / \mathrm{min} / \mathrm{mg}$ protein. CAT activity in the hippocampus was measured at $37{ }^{\circ} \mathrm{C}$ by following the rate of disappearance of $\mathrm{H}_{2} \mathrm{O}_{2}$ at $240 \mathrm{~nm}$ [30]. To initiate the CAT activity, $0.2 \mathrm{ml}$ of tissue homogenate $/ 0.2 \mathrm{ml}$ of serum was added to the reaction mixture containing $1.9 \mathrm{~mL}$ of $100 \mathrm{mM}$ Phosphate buffer ( $\mathrm{pH} 7.0$ ), $0.1 \mathrm{~mL}$ of enzyme extract, and $1.0 \mathrm{~mL}$ of $0.075 \% \mathrm{H}_{2} \mathrm{O}_{2}$ solution. CAT activity was assayed in $1.0 \mathrm{~min}$ at $240 \mathrm{~nm}$. GSH-Px activity in the brain was estimated according to the method described by Ellman [31]. The reaction mixture contained $0.1 \mathrm{M}$ phosphate buffer ( $\mathrm{pH}$ 7.0), $1 \mathrm{mM}$ EDTA, $10 \mathrm{mM}$ glutathione (GSH), $1 \mathrm{mM} \mathrm{NaN3}, 1$ unit of glutathione reductase, $1.5 \mathrm{mM} \mathrm{NADPH}$ and $0.1 \mathrm{ml}$ of homogenate/serum. After incubation for $10 \mathrm{~min}$ at $37 \mathrm{o} \mathrm{C}, \mathrm{H} 2 \mathrm{O} 2$ was added to each sample at a final concentration of $1 \mathrm{mM}$. GPX activity was measured as the rate of NADPH oxidation at $340 \mathrm{~nm}$. The MDA content, a quantitative measurement of lipid peroxidation, was assayed in the form of thiobarbituric acid reactive substances by the method of Wills [32]. Collected supernatant was incubated in a solution containing $8.1 \%$ SDS, $20 \%$ acetic acid (pH 3.5), $0.8 \%$ TBA (Thiobarbituric acid) for $1 \mathrm{~h}$ at $95{ }^{\circ} \mathrm{C}$. After cooling at RT, the samples were well mixed with butanol/pyridine mixture $(15: 1, \mathrm{v} / \mathrm{v})$, and the aqueous phase was carefully collected by aspiration. Absorbance of the aqueous phase was found out at $532 \mathrm{~nm}$. MDA content was expressed as $\mathrm{nmol} / \mathrm{mg}$ protein. Advanced protein oxidation product (APOP) was estimated by the method followed by Salde et al. [33]. Briefly, two $\mathrm{mL}$ of plasma was diluted $1: 5$ in PBS: $0.1 \mathrm{~mL}$ of $1.16 \mathrm{M}$ potassium iodide was then added to each tube, followed by $0.2 \mathrm{~mL}$ acetic acid after $2 \mathrm{~min}$. The absorbance of the reaction mixture was immediately read at $340 \mathrm{~nm}$ against a blank containing $2 \mathrm{~mL}$ of PBS, $0.1 \mathrm{~mL}$ of $\mathrm{KI}$, and $0.2 \mathrm{~mL}$ of acetic acid. The chloramine-T absorbance at $340 \mathrm{~nm}$ being linear within the range of $0-100 \mathrm{mmol} / \mathrm{L}$, AOPP concentrations were expressed as $\mu \mathrm{mol} \cdot \mathrm{L}^{-1}$ chloramine$\mathrm{T}$ equivalents The accumulation of nitrite, an indicator of nitric oxide (NO) production was determined by a colorimetric assay as described by Green et al. [34]. Aliquots of $50 \mu \mathrm{L}$ of supernatants were incubated with $50 \mu \mathrm{L}$ of Griess reagent, consisting of $1 \%$ sulfanilamide, $0.1 \% \mathrm{~N}$-(1-naphthylethylenediamine) dihydrochloride and $2.5 \%$ ortho-phosphoric acid. After $10 \mathrm{~min}$ at room temperature, the optical density was measured at $540 \mathrm{~nm}$. Nitrite concentrations were calculated by comparison to a calibration curve obtained with sodium nitrite standard solutions.

\section{Statistical analysis}

Data is expressed as means \pm SEM. All data are presented as the mean \pm SD of five animals. Data was analyzed with One Way Analysis of Variance (ANOVA) using a statistical software package (SPSS for Windows, version 21, IBM Corporation, NY, USA) followed by Tukey's-HSD multiple range post-hoc test. Values were considered significantly different at $p<0.05$.

\section{Results}

\section{Neuropharmacological effect of LM Open field test}

In the experiment, the extract showed a noticeable decrease in locomotion in the test animals from the second observation period to last study period at both dose levels. The depressant action was increased with time and a noticeable result was found at $120 \mathrm{~min}$ of test sample administration. Test animals showed significant decrease in number of movements at the dosages of 100 and $200 \mathrm{mg} / \mathrm{kg}(10 \pm 2.83,8 \pm 2.12$, respectively) as compared to $13 \pm 1.41$ in the NC group at $120 \mathrm{~min}$ of administration of LM). Figure 2 shows the dose dependent CNS depressant activities which were statistically significant $(p<0.05)$ compared to NC and PC groups.

\section{Hole cross test}

In hole cross test, the animal treated with LM showed dose dependent reduction in the locomotor activity. At LM 200, number of holes crossed $(1.5 \pm 0.71)$ was comparable with that of standard drug diazepam $(1.5 \pm 0.71)$. The extract 


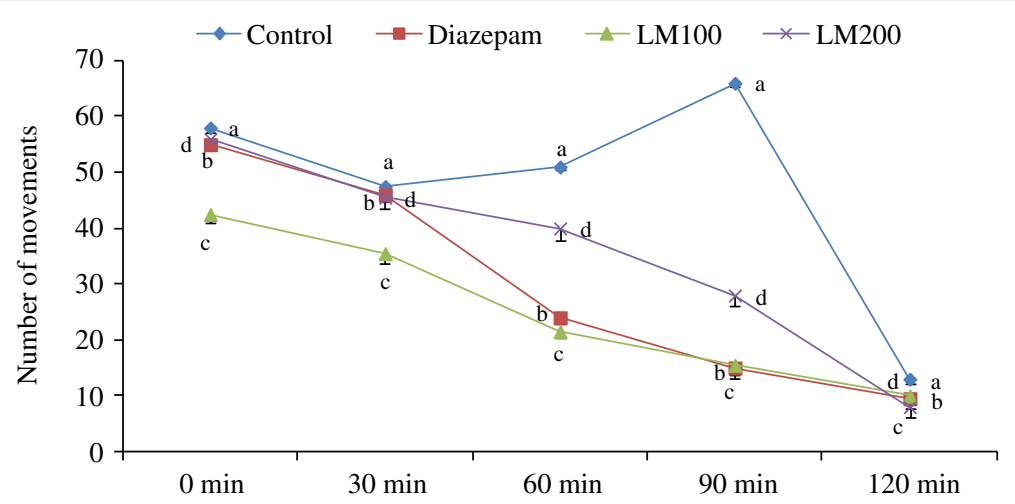

Fig. 2 Effect of LM on locomotor activity in open field test for Wistar albino rats. Data are shown as Mean \pm SD for triplicate. Data were analyzed by one-way ANOVA followed by Tukey's post hoc test (SPSS, Version 21.0,NY) for multiple comparisons. abcdValues with different superscript letters over the lines for a given parameter are significantly different from each other group of animals. Values were considered as significant with $p<0.05$

produced reduction in spontaneous motor activity, and this effect may be attributed to CNS depression of standard drug diazepam $(1.5 \pm 0.71)$. LM100 also reduced the locomotor effect but the values were not significant compared to PC group. The CNS was found to be depressed through the observation period 0 to $120 \mathrm{~min}$ (Fig. 3).

\section{EPM test}

LM at both the doses increased the percentage of entries into the open arms (Fig. 4). The percentage of time spent into open arms also increased at the LM200 mg/ $\mathrm{kg}$ doses $(16 \pm 0.08)$ as compared to the $\mathrm{NC}$ group $(10.51 \pm 1.72)$. There was also a significant increase in duration of time $(42 \pm 2.83)$ for LM200, into open arm, which was statistically significant as compared to that $(35.5 \pm 3.54)$ of reference group.

\section{Thiopental sodium induced sleeping time test}

Thiopental sodium induced sleeping time test revealed that both the doses induced sleep at a rapid stage as compared to NC. In addition, both doses of LM prolonged the duration of sleeping time $(115 \pm 10.60$, $142 \pm 10.60$ respectively) in comparison to NC (100 \pm 14.14). As test sample decreased latent period (onset of sleep) and increased the duration of sleep, the LM showed dose dependent sedative effects which were statistically significant $(p<0.05)$. Figures 5 and 6 represent the activity in thiopental sodium induced sleeping time test.

\section{Effect of LM on diazepam-induced amnesia}

The mean escape latency for the trained rats decreased during the course of the 5-day trials. There was no noticeable difference found in the mean escape latency between any two groups on the first, second and third day. However, on the fourth and fifth day, the mean escape latency of the PC group was longer than that of the control group $(P<0.01)$. Compared with the $\mathrm{PC}$ group, the escape latency of rats treated with LM decreased to a prominent degree $(P<0.01$, Fig. 7$)$. At the last day of trial, mean escape latency of LM200 was $2.41 \pm 0.32$, which was less than the $\mathrm{NC}$ and $\mathrm{PC}$ group $(3.25 \pm 0.44$

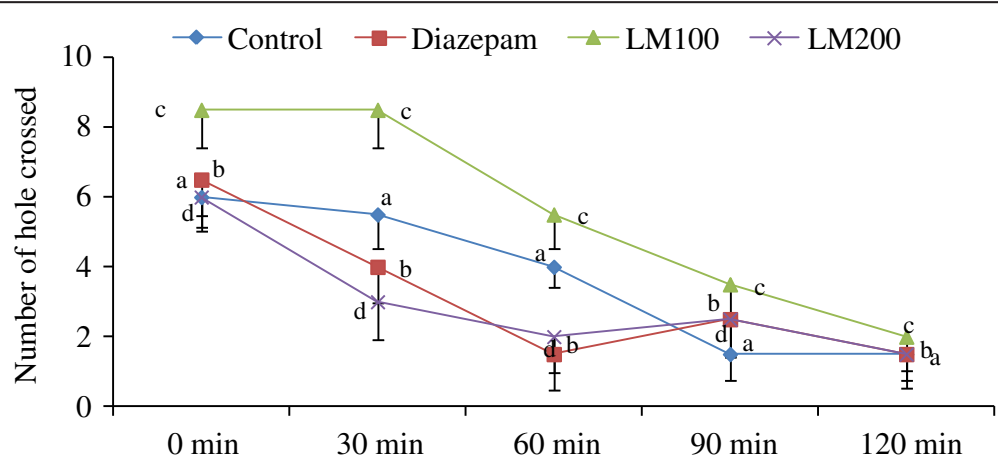

Fig. 3 Effect of LM on locomotor activity in hole-cross test for Wistar albino rats. Data are shown as Mean \pm SD for triplicate. Data were analyzed by one-way ANOVA followed by Tukey's post hoc test (SPSS, Version 21.0, NY) for multiple comparisons. abcdValues with different superscript letters over the lines for a given parameter are significantly different from each other group of animals. Values were considered as significant with $p<0.05$ 


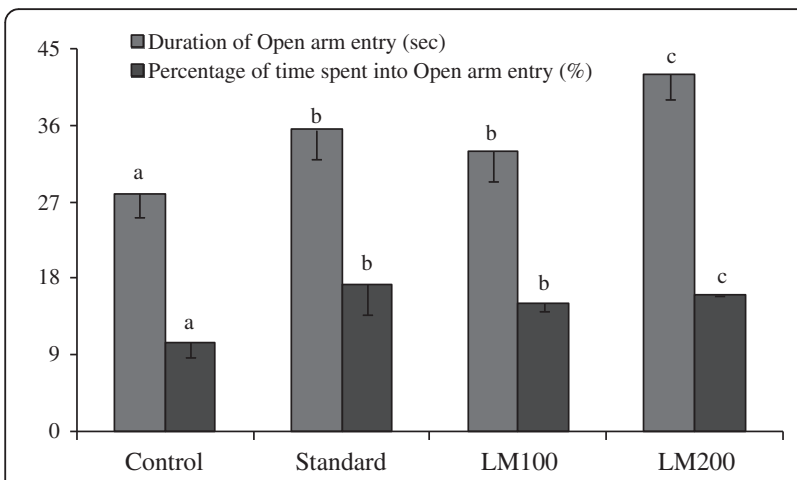

Fig. 4 Effect of LM on duration of open arm in elevated plus maze (EPM) test for Wistar albino rats. Data are shown as Mean \pm SD for triplicate. Data were analyzed by one-way ANOVA followed by Tukey's post hoc test (SPSS, Version 21.0,NY) for multiple comparisons.

abcdValues with different superscript letters over the bars for given parameters are significantly different from each other group of animals. Values were considered as significant with $p<0.05$

and $2.96 \pm 0.76$, respectively). In the probe trial study, the time spent in the target quadrant was significantly shorter in PC group as compared to that in the NC group. The rats in the LM groups spent more time in the target quadrant than the PC group (Fig. 8).

\section{Effect of LM on SOD, CAT, Glutathione peroxidase, MDA,} AOPP, NO activity in hippocampus

Compared with the NC group, SOD, catalase and GSHPx activities were significantly decreased following diazepam administration. Treatment with LM alleviated the changes of SOD, catalase and GSH-Px activities Fig. 9 (a-c). In comparison with the NC group, the MDA content in diazepam-treated mice increased. However, LM significantly attenuated the increase of MDA content induced by diazepam (Fig. 8d). In case of AOPP, LM has

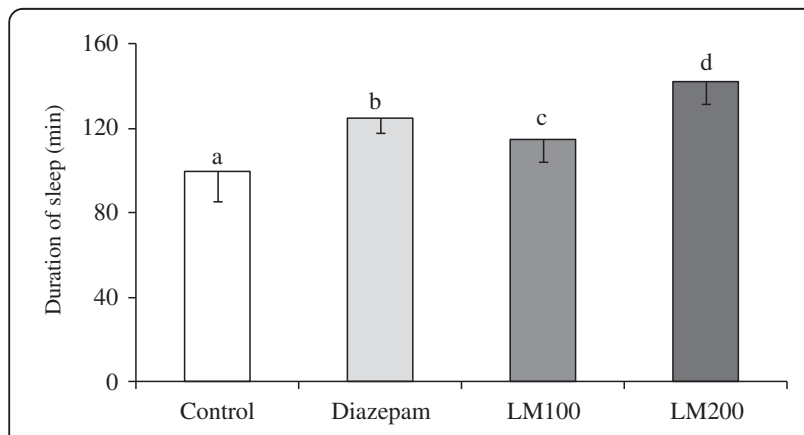

Fig. 5 Effect of Leea macrophylla on duration of sleep in thiopental sodium induced sleeping time test for Wistar albino rats. Data are shown as Mean \pm SD for triplicate. Data were analyzed by one-way ANOVA followed by Tukey's post hoc test (SPSS, Version 21.0,NY) for

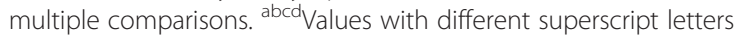
over the bars for a given parameter are significantly different from each other group of animals. Values were considered as significant with $p<0.05$

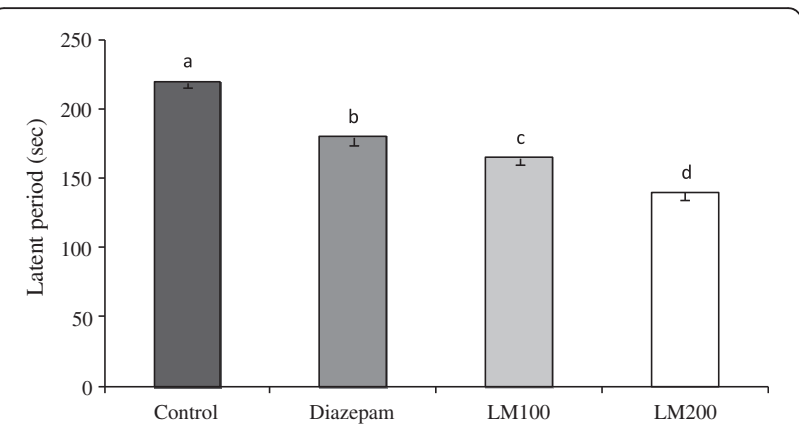

Fig. 6 Effect of Leea macrophylla on latent period in thiopental sodium induced sleeping time test for Wistar albino rats. Data are shown as Mean \pm SD for triplicate. Data were analyzed by one-way ANOVA followed by Tukey's post hoc test (SPSS, Version

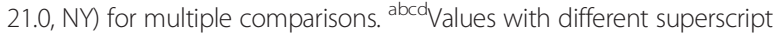
letters over the bars for a given parameter are significantly different from each other group of animals. Values were considered as significant with $p<0.05$

dose-dependently decreased AOPP in test groups. However, LM200 decreased the content of AOPP at value of $55.75 \pm 7.42$ which was statistically significant $(p<0.05)$ to PC group. Level of nitric oxide in $\mathrm{NC}$ group is $4.22 \pm$ 1.61. LM200 as well significantly decreased nitric oxide level (Figs. 10 and 11).

\section{Discussion}

In our present study neuropharmacological effects of LM have been evaluated through open field test, holecross test, EPM and thiopental sodium induced sleeping time test on Wistar albino rats. The most important step in evaluating drug action on the CNS is to observe the behavior of the test animals [35]. Open field test and hole-cross tests were carried out to evaluate locomotor activity of animals. Increase in locomotor effect is considered as an increase in alertness and decrease in locomotor activity indicated sedative effect [36].

Present study revealed that the LM showed dose dependent CNS depressant activities. In Open field test, both LM100 and LM200 showed a noticeable decrease in locomotion in the test animals from the second observation period to the end of study period. In hole-cross test, LM showed a dose dependent reduction in the locomotor activity. But maximum locomotor reduction was shown by LM200.

Different anxiolytic, sedative-hypnotic drugs are elucidated their action through Gamma-amino-butyric acid (GABA). GABA subtype A (GABAA) is the major inhibitory neurotransmitter in the central nervous system [37]. Therefore it is possible that, this extract may act by potentiating GABAergic inhibition in the CNS via membrane hyperpolarization which leads to a decrease in the firing rate of critical neurons in the brain. It may also be due to direct activation of GABA receptor by the extract 


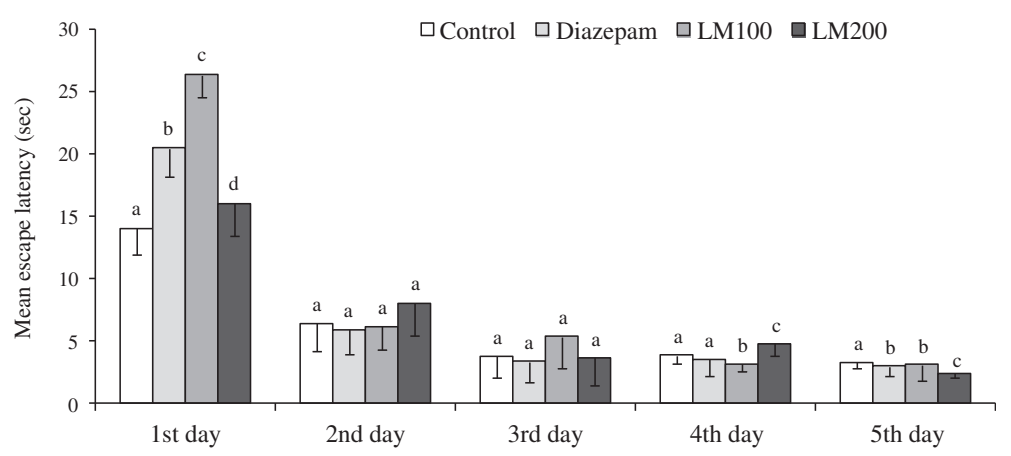

Fig. 7 Effect of Leea macrophylla on mean escape latency in acquisition trial for Wistar albino rats. Data are shown as Mean \pm SD for triplicate. Data were analyzed by one-way ANOVA followed by Tukey's post hoc test (SPSS, Version 21.0,NY) for multiple comparisons. ${ }^{\text {abcd } V a l u e s ~ w i t h ~}$ different superscript letters over the bars for a given parameter are significantly different from each other group of animals. Values were considered as significant with $p<0.05$

[38]. As LM decreased locomotor activity in animals, it indicates CNS depressant activity.

Anxiolytic effect of drug molecules is evaluated by EPM test which is a well-established model for assessing neuropharmacological responses of rodents [39]. The effect is observed when the experimental drug increases open arms entries without altering the total number of arm entries. In our present study, both of the doses of LM increased the percentage of entries of rats into the open arms. But LM200 showed a significant increase in the percentage of time spent in the open arms of the maze. This was slightly larger than the effects observed following treatment with the reference anxiolytic drug diazepam. These results indicate an anxiolytic-like activity of LM.

Thiopental at appropriate dose induces hypnosis by potentiating GABA mediated postsynaptic inhibition through allosteric modification of GABA A type
(GABAA) receptors. In the current research, both LM100 and LM200 potentiated sleep induced by thiopental suggesting that the roots of the plant possess a sleep inducing property. Additionally, substances which possess CNS depressant activity either decrease the time for onset of sleep or prolong the duration of sleep or both [40]. This is consistent with our study where LM100 and LM200 significantly increased the duration of sleep. Similar findings were recorded by Fujimori and Cobb [21] who proposed that the enhancement of hypnosis is a good index of CNS depressant activity mediated by GABAA receptor (as described earlier). The receptor complex is involved in various neuropsychological disorders such as epilepsy, depression, Parkinson's and Alzheimer's disease [41]. Many flavonoids such as isoflavones S-(-)-equol, 4'-hydroxy-7-methoxyisoflavone and 3',7-dihydroxyisoflavone, were found to be ligands for GABAA receptors in the CNS; which led to the hypothesis that they act as

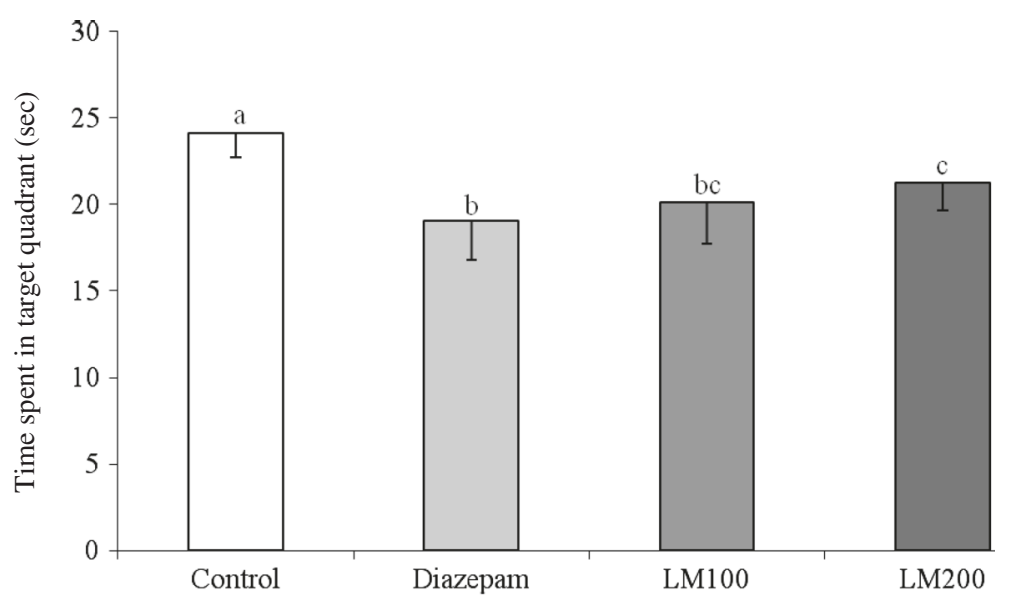

Fig. 8 Effect of Leea macrophylla on time spent in target quadrant in retrieval trial for Wistar albino rats. Data are shown as Mean \pm SD for triplicate.

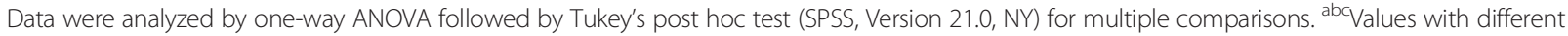
superscript letters over the bars for a given parameter are significantly different from each other group of animals. Values were considered as significant with $p<0.05$ 
(a)

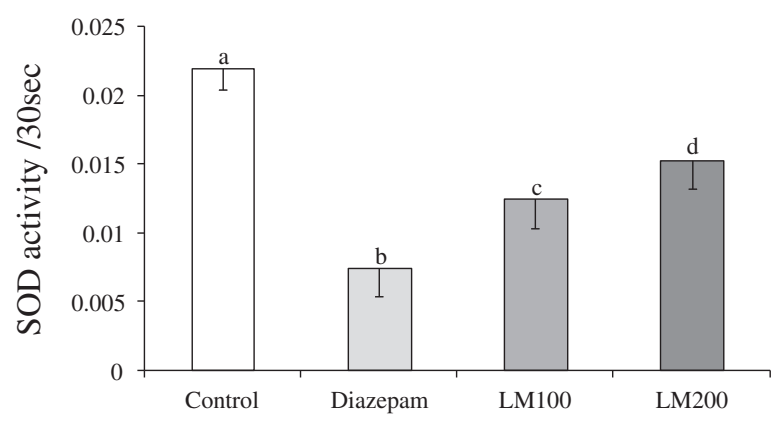

(c)

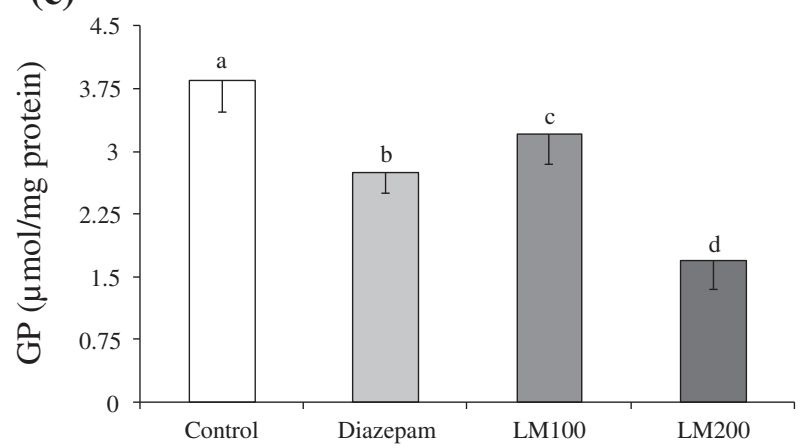

(b)

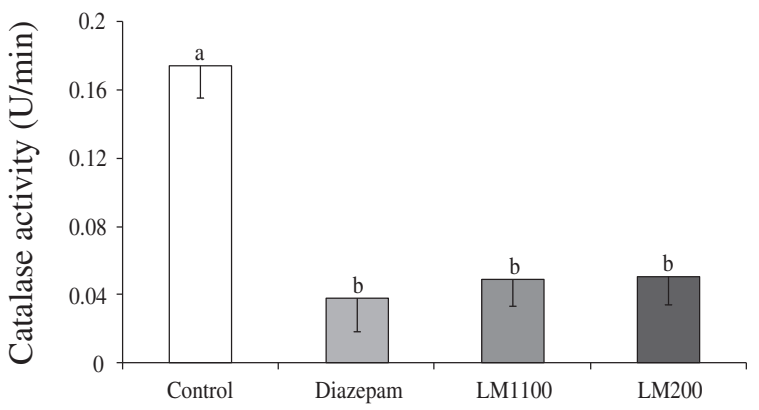

(d)

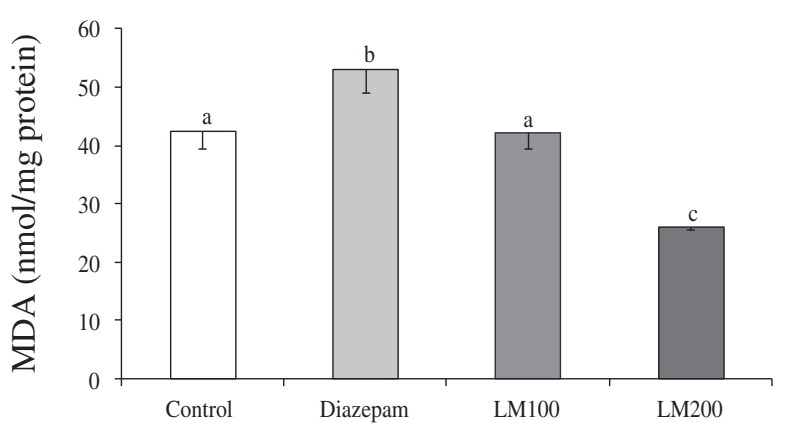

Fig. 9 Effect of Leea macrophylla on superoxide dismutase (SOD) activity (a); catalase activity (b); Glutathione peroxidase (GP) (c); and Malonaldehyde (MDA) (d) in hippocampus for Wistar albino rats. Data are shown as Mean \pm SD for triplicate. Data were analyzed by one-way ANOVA followed by

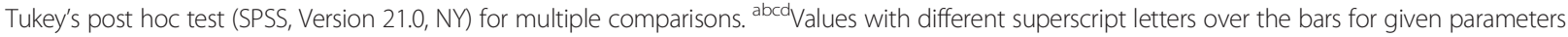
are significantly different from each other group of animals. Values were considered as significant with $p<0.05$

benzodiazepine-like molecules [42, 43]. This is supported by their behavioral effects in animal models of anxiety, sedation and convulsion [3]. Since Leea macrophylla was found to possess flavonoids, alkaloids, tannins and terpenoids, behavioral effects in animal models of anxiety and sedation could be supported by different doses LM.

Benzodiazepines (BZs) induce anterograde amnesia in both human and animals [44]. In this research, the effect of LM on diazepam induced amnesia was investigated using Morris water maze scale for behavioral experiments. Morris water maze is used to test spatial memory of the rats [45]. It is most widely used tasks in behavioral neuroscience for studying psychological processes. In our study, the animals in the diazepam group took a significantly longer time to locate the hidden platform on days 4 and 5 , which indicated

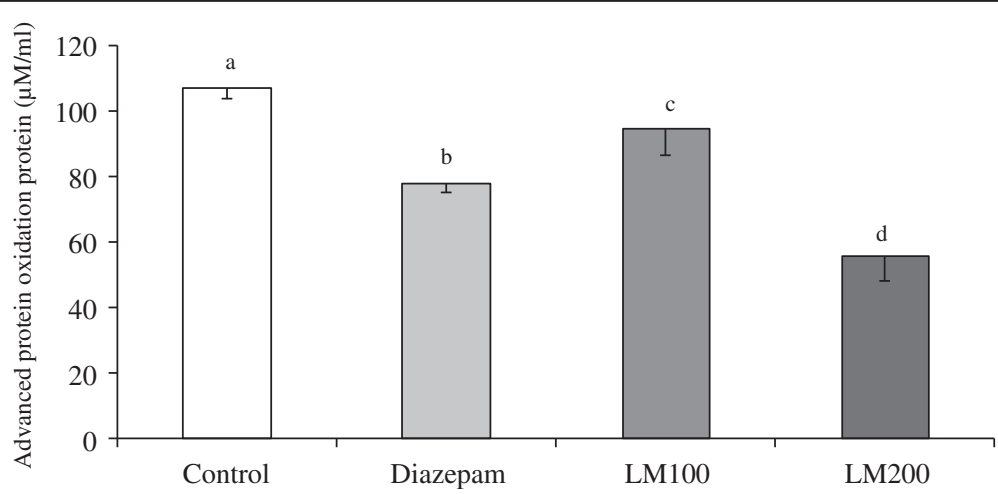

Fig. 10 Effect of Leea macrophylla on advanced oxidation protein product (AOPP) in hippocampus for Wistar albino rats. Data are shown as Mean \pm SD for triplicate. Data were analyzed by one-way ANOVA followed by Tukey's post hoc test (SPSS, Version 21.0, NY) for multiple comparisons. ${ }^{\text {abcdValues }}$ with different superscript letters over the bars for a given parameter are significantly different from each other group of animals. Values were considered as significant with $p<0.05$ 


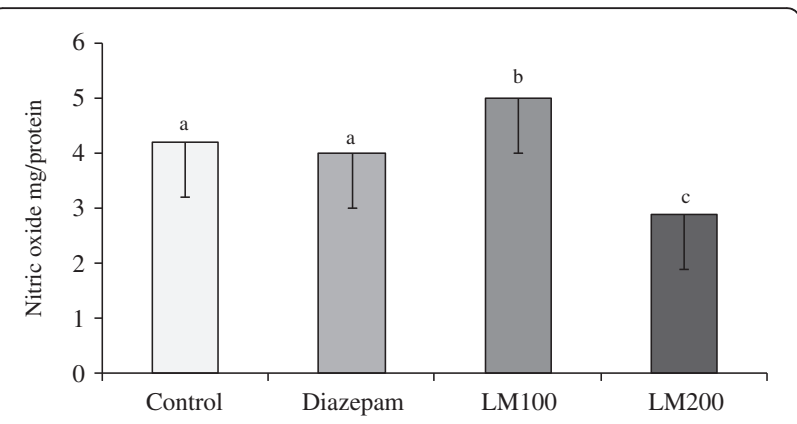

Fig. 11 Effect of Leea macrophylla on nitric oxide (NO) in hippocampus for Wistar albino rats. Data are shown as Mean \pm SD for triplicate. Data were analyzed by one-way ANOVA followed by Tukey's post hoc test (SPSS, Version 21.0, NY) for multiple comparisons. ${ }^{a b c}$ Values with different superscript letters over the bars for a given parameter are significantly different from each other group of animals. Values were considered as significant with $p<0.05$

that diazepam administration impaired spatial learning. By day 6, we assessed memory retention in a probe trial which involved removing the hidden platform from the pool and measuring the length of time the animals spent in the target quadrant. The probe trial revealed that diazepam-treated rats spent significantly less time swimming in the target quadrant. Therefore, the study showed that diazepam resulted in long lasting spatial memory deficits as well [46]. On the contrast, LM attenuated the spatial learning and memory impairments as compared to animals treated by diazepam alone. These findings suggested that co-administration of LM with diazepam significantly ameliorated diazepam-induced anterograde amnesia. It is reasonable to speculate that this effect is being mediated by the GABAergic system. The molecular events underlying the antiamnesic effect of LM need to be discerned to not only screen the active constituents of Leea macrophylla but can facilitate development of novel targets to enhance memory [47].

Free radicals and related molecules are often classified together as reactive oxygen species (ROS). The formation of ROS in nerve cells are numerous, therefore, cells have to maintain an effective antioxidant system in order to protect themselves against ROS overload and subsequent damage [48]. During oxidative stress, ROS production increases and surpasses the capacity of endogenous free radical scavengers such as SOD, catalase and GSH-Px. In addition, the brain is especially sensitive to oxidative stress because it utilizes high levels of oxygen and contains large amounts of easily peroxidizable fatty acids. It was also reported that formation of ROS was involved in neurotoxicity of many xenobiotics [49]. In this research, diazepam caused a significant change in MDA, NO, AOPP content and SOD, catalase and GSH-Px activities in hippocampus. However, co-administration of LM with diazepam not only reduced MDA content but also increased SOD, catalase and GSH-Px activities in hippocampus of rats. The observed effect may be lying in the radical scavenging properties of LM containing polyphenolics which are established for their antioxidative nature. The present findings, therefore, demonstrated the correlation between antiamnesic effects of LM against diazepaminduced amnesia and its antioxidative capacity [50], but the causal relationship remains to be determined in the future work.

\section{Conclusion}

The results of this study provided a scientific support for the use of LM in the neurological disorders. It also revealed the CNS depressant, anxiolytic and sedative effects of LM which ameliorated diazepam-induced amnesia in animal models. It has significantly increased antioxidative enzymes superoxide dismutase, catalase and glutathione peroxidase in the hippocampus of brain. It also decreases oxidative biomarkers such as lipid peroxidation, nitric oxide and advanced oxidation protein product. Therefore, the use of this plant in the reduction of oxidative stress associated - neurodegeneration is become evident by this study although further investigation of the chemical constituents of this plant is necessary to justify their involvement in the observed actions.

\section{Source of support}

Authors wish to thank University of Chittagong to allocate a fund for conducting this research under a UGC-Dean research grant (Ref.5448/ Res/Plan/Pub/CU/2013).

\section{Authors' contribution}

MAR and JA have planned and designed the research. SF has collected sample, performed the experimental works in laboratory, analyzed the data and written the manuscript. MAR has also endeavored data analysis, interpretation of the results, revision of the whole manuscript with grammatical checking and technical design. MMAA and JMKHC have assisted the laboratory analysis of the work. All authors read and approved the final manuscript.

\section{Competing interest}

The authors declare that they have no competing interest.

\section{Author details}

'Department of Biochemistry \& Molecular Biology, University of Chittagong, Chittagong 4331, Bangladesh. ${ }^{2}$ Department of Pharmacy, North South University, Bashundhara, Dhaka 1229, Bangladesh.

Received: 7 June 2016 Accepted: 12 August 2016

Published online: 16 August 2016

\section{References}

1. Simpson T, Pase M, Stough C. Bacopa monnieri as an antioxidant therapy to reduce oxidative sress in the aging brain. Evid Based Complement Alternat Med. 2015;2015:615384.

2. Wang Z, Wan H, Li J, Zhang H, Tian M. Molecular imaging in traditional Chinese medicine therapy for neurological diseases. BioMed Res Int. 2013; 2013:608430.

3. Kales A, Bixler E, Vela-Bueno A, Soldatos CR, Manfredi RL. Alprazolam: effects on sleep and withdrawal phenomena. J Clin Pharmacol. 1987;27:508-15.

4. Ernst E. Herb-drug interactions: potentially important but woefully under-researched. Eur J Clin Pharmacol. 2000;56:523-4. 
5. Marder M, Paladini AC. GABA-A receptor ligands of flavonoid structure. Curr Top Med Chem. 2002;2:853-67.

6. Singh RS, Singh AN. On the identity and economic-medical uses of Hastikarnapalsa (Leea macrophylla Roxb.) as evinced in the ancient texts and traditions. Indian J Hist Sci. 1981;16:219-22.

7. Ahmed ZU, Begum ZNT, Hassan MA. Encyclopedia of Flora and Fauna of Bangladesh: Index Volume-Flora, vol. 13. 1st ed. Dhaka: Asiatic Society of Bangladesh; 2009.

8. Choudhary K, Singh M, Pillai U. Ethnobotanical survey of Rajasthan - an update. Am-Eur J Bot. 2008;1:38-45

9. Uddin SN. Traditional uses of ethno-medicinal plants of the Chittagong Hill Tracts. Bangladesh National Herbarium, Dhaka, Bangladesh, First Edition. 2006.

10. Nizami AN, Rahman MA, Ahmed NU, Islam MS. Whole Leea macrophylla ethanolic extract normalizes kidney deposits and recovers renal impairments in an ethylene glycol-induced urolithiasis model of rats. Asian Pac J Trop Med. 2012;5:533-8.

11. Akhter S, Rahman MA, Aklima J, Hasan MR, Chowdhury JMKH. Antioxidative role of Hatikana (Leea macrophylla Roxb.) partially improves the hepatic damage induced by $\mathrm{CCl}_{4}$ in Wistar Albino rats. BioMed Res Int. 2015; 2015:ID 356729. doi:10.1155/2015/356729.

12. Islam MB, Sarkar MMH, Shafique MZ, Jalil MA, Haque MZ, Amin R. Phytochemical screening and anti-microbial activity studies on Leea macrophylla seed extracts. J Sci Res. 2013;5:399-405.

13. Zaoui A, Cherrah Y, Mahassini N, Alaoui K, Amarouch H, Hassar M. Acute and chronic toxicity of Nigella sativa fixed oil. Phytomedicine. 2002;9:69-74.

14. Parasuraman S. Toxicological screening. J Pharmacol Pharmacother. 2011; 2(2):74-9.

15. Litchfield JT, Wilcoxon F. A simplified method of evaluating dose effect experiments. J Pharmacol Exp Ther. 1949;96:99-133.

16. Joshi H, Megeri K. Antiamnesic evaluation of C. phlomidis Linn. bark extract in mice. Braz J Pharm Sci. 2008;44(4):out./dez.

17. Joshi H, Parle M. Evaluation of the antiamnesic effects of Phyllanthus amarusin mice. Colomb Med. 2007:38(2):132-9.

18. Gupta BD, Dandiya PC, Gupta ML. A psychopharmacological analysis of behavior in rat. Jpn J Pharmacol. 1971:21:293.

19. Takagi K, Watanabe M, Saito H. Studies on the spontaneous movement of animals by the hole cross test: effect of 2-dimethylaminoethane. Its acylates on the central nervous system. Jpn J Pharmacol. 1971;21:797.

20. Walf AA, Fry CA. The use of the elevated plus maze as an assay of anxietyrelated behavior in rodents. Nat Protoc. 2007;2:322-8.

21. Fujimori $H$, Cobb D. Central nervous system depressant activity of MA1337 7,3 (4-M-Chlorophenyl -1- piperazyl)-2,4, (14, 34) Quinazolinedione hydrochloride. Psycho-pharmacol. 1965;7:374-7.

22. Wang T, Shan SY, Han B, Zhang LM, Fu FH. Salvianolic acid A exerts antiamnesic effect on diazepam-induced anterograde amnesia in mice. Neurochem Res. 2011;36:103-8.

23. Martínez-Ferrer A, Boyano-Adánez MC, Izquierdo-Claros RM, Arilla-Ferreiro E. Diazepam attenuation of somatostatin binding and effect of somatostatin on accumulation of inositol 1,4,5-trisphosphate in the rat frontoparietal cortex. Neuropsychopharmacology. 2000;23:178-87.

24. Kesner RP, Exans RB, Hunt MA. Further evidence in support of the neurological basis of an attribute model of memory: role of hippocampus. Int J Neurosci. 1987:21-22:184-96

25. Nunez J. Morris Water Maze Experiment. J Vis Exp. 2008;19:897.

26. D'Hooge R, De Deyn PP. Applications of the Morris water maze in the study of learning and memory. Brain Res Rev. 2001:36:60-90.

27. Anand A, Saraf MK, Prabhakar S. Sustained inhibition of brotizolam induced anterograde amnesia by norharmane and retrograde amnesia by l-glutamic acid in mice. Behav Brain Res. 2007;182:12-20.

28. Malleret G, Hen R, Guillou JL, Segu L, Buhot MC. 5-HT1B receptor knock-out mice exhibit increased exploratory activity and enhanced spatial memory performance in the Morris water maze. J Neurosci. 1999:19:6157-68.

29. Misra HP, Fridovich I. The role of superoxide anion in the autoxidation of epinephrine and a simple assay for superoxide dismutase. J Biol Chem. 1972;247:3170-5

30. Aebi H. Catalase in vitro. Methods Enzymol. 1984;105:121-6.

31. Ellman GL. Tissue sulfhydryl groups. Arch Biochem Biophys. 1959;82:70-7.

32. Wills ED. Mechanism of lipid peroxide formation in animal. Biochem J. 1966; 99:667-76.
33. Salde CV, Albesa I. Reactive oxidant species and oxidation of protein and haemoglobin as biomarkers of susceptibility to stress caused by chloramphenicol. Biomed Pharmacother. 2009;63:100-4.

34. Green LC, Wagner DA, Glogowski J, Skipper PL, Wishnok JS, Tannenbaum SR. Analysis of nitrate, nitrite, and [15N] nitrate in biological fluids. Anal Biochem. 1982;126:131-8.

35. Howlader SI, Dey SK, Hira A, Ahmed A, Hossain H. Antinociceptive and neuropharmacological activities of ethanolic extract of the fruits of Ficus hispida Linn. Afr J Pharm Pharmacol. 2012;6:2837-44.

36. Verma A, Jana GK, Sen S, Chakraborty R, Sachan S, Mishra A. Pharmacological evaluation of Saraca indica leaves for central nervous system depressant activity in mice. J Pharm Sci Res. 2010;2:338-43.

37. Mamun A, Khatun H, Islam MR, Nahar L, Doha KMSU, Ripa FA. Evaluation of CNS depressant and analgesic activities of the methanol extract of Piper longum linn. Leaves. Int J Pharm Sci Res. 2011;2:2874-9.

38. Kolawole OT, Makinde JM, Olajide OA. Central nervous depressant activity of Russelia equisetiformis. Niger J Physiol Sci. 2007;22:59-63.

39. Dawson GR, Tricklebank MD. Use of the elevated plus maze in the search for novel anxiolytic agents. Trends Pharmacol Sci. 1995;16:33-6.

40. Raquibul Hasan SM, Hossain MM, Akter R, Jamila M, Mazumder EH, Rahman S. Sedative and anxiolytic effects of different fractions of the Commelina benghalensis Linn. Drug Discov Ther. 2009:3:221-7.

41. Weinreb $O$, Mandel S, Amit T, Youdim MB. Neurological mechanisms of green tea polyphenols in Alzheimer's and Parkinson's diseases. J Nutr Biochem. 2004; 15:506-16.

42. Hanrahan JR, Chebib M, Johnston GAR. Flavonoid modulation of GABAA receptors. Br J Pharmacol. 2011;163:234-45.

43. Rudi DH, Peter PD. Applications of Morris water maze in the study of learning and memory. Brain Res Rev. 2001;36:60-90.

44. Lister RG. The use of plus maze to measure anxiety in mouse. Psychpharmacol. 1987:92:180-5.

45. Morris RGM, Garrud P, Rawlins JN, O'Keefe J. Place navigation impaired in rats with hippocampal lesions. Nature. 1982:297:681-3.

46. Prabhakar S, Saraf MK, Pandhi P, Anand A. Bacopa monniera exerts antiamnesic effect on diazepam-induced anterograde amnesia in mice. Psychopharmacol (Berl). 2008;200:27-37.

47. Kim DH, Jeon SJ, Son KH, Jung JW, Lee S, Yoon BH, Lee JJ, Cho YW, Cheong $\mathrm{JH}, \mathrm{Ko} \mathrm{KH}, \mathrm{Ryu} \mathrm{JH}$. The ameliorating effect of oroxylin A on scopolamine induced memory impairment in mice. Neurobiol Learn Mem. 2007:87:536-46.

48. Behl C, Moosmann B. Antioxidant neuroprotection in Alzheimer's disease as preventive and therapeutic approach. Free Radic Biol Med. 2002;33:182-91.

49. Ravindranath $\vee$, Boyd MR. Xenobiotic metabolism in brain. Drug Metab Rev. 1995;27:419-48

50. Musavi S, Kakkar P. Pro and antioxidant responses to repeated administration of diazepam in rat brain. Mol Cell Biochem. 2000;206:97-103.

\section{Submit your manuscript to a SpringerOpen ${ }^{\circ}$ journal and benefit from:}

- Convenient online submission

- Rigorous peer review

- Immediate publication on acceptance

- Open access: articles freely available online

- High visibility within the field

- Retaining the copyright to your article

Submit your next manuscript at $>$ springeropen.com 